\title{
Compressibility of multicomponent, charged model biomembranes tunes permeation of cationic nanoparticles
}

Anurag Chaudhury, $\dagger, \S$ Gopal Kishor Varshney, $\dagger, \S$ Koushik Debnath, $\$$ Gangadhar Das, If Nikhil

R. Jana, $\$$ and Jaydeep Kumar Basu*, $\uparrow$

$\dagger$ Department of Physics, Indian Institute of Science, Bangalore 560012, India

$\ddagger$ School of Materials Science, Indian Association for the Cultivation of Sciences, Kolkata 700032,

India

IIKEK-High Energy Accelerator Research Organization, 1-1 Oho, Tsukuba, Ibaraki 305-0801, Japan

$\S$ Contributed equally to this work

E-mail: basu@iisc.ac.in

Phone: +91-80-2293 3281 


\section{Fabrication of two-component supported lipid bilayers:}

Langmuir-Blodgett/Langmuir-Schaefer (LB/LS) method has been utilized for the deposition of two-component lipid bilayers on silicon substrate. Prior to transfer of bilayer, silicon substrates were made hydrophilic by boiling in a solution of $\mathrm{H}_{2} \mathrm{O}: \mathrm{NH}_{4} \mathrm{OH}: \mathrm{H}_{2} \mathrm{O}_{2}=5: 1: 1$ for 10 minutes followed by subsequent rinsing in deionized water several times. A chloroform solution of mixed phospholipids was spread uniformly over the water surface by using a Hamilton syringe. After spreading, the barriers were kept stationary for 15 minutes for complete evaporation of chloroform. The temperature of trough was kept at $20{ }^{\circ} \mathrm{C}$. Surface pressure versus area/molecule isotherms were recorded using the KSV LB rectangular mini Trough (area, $243 \mathrm{~cm}^{2}$ ) equipped with a wilhelmy balance. A platinum sensor of accuracy $0.1 \mathrm{mN} / \mathrm{m}$ was used to measure the interfacial surface pressure. The barriers were then compressed at a constant rate of $5 \mathrm{~mm} / \mathrm{min}$ to record the isotherms of the monolayers. Fig. 1(a) shows the pressure-area isotherm of these two-component monolayers. Supported lipid bilayers (SLBs) were formed upon controlled transfer of lipid interfacial monolayers onto pretreated silicon substrate. Multiple compression-expansion cycles were carried out during the preparation of bilayers and subsequently they were transferred at a surface pressure of $32 \mathrm{mN} / \mathrm{m}$ to the hydrolyzed silicon substrate. The first monolayer was transferred by vertical withdrawal of the substrate at a speed of $5 \mathrm{~mm} / \mathrm{min}$. In the monolayer transferred onto the hydrophilic silicon surface, the charged head groups of lipid faced the silicon surface and the hydrophobic hydrocarbon chains were directed towards the air. The second monolayer was transferred at the same surface pressure by a horizontal down stroke on the substrate. After transfer, the SLBs were moved to a container under water and stored at room temperature for further use. 
The isothermal compression modulus $(\beta)$ of the lipid monolayer is calculated by using the equation,

$$
\beta=-A\left(\frac{\partial A}{\partial \pi}\right)_{T}
$$

Where, $\pi$ and $A$ are the measured surface pressure and area per molecule, respectively, at a constant temperature, $\mathrm{T}=20{ }^{\circ} \mathrm{C}$. The parameter $\left(\frac{\partial A}{\partial \pi}\right)_{T}$ is calculated by differentiating the pressure-area isotherm. The isothermal compression modulus used to describe mechanical properties of these monolayers. In addition, higher $\beta$ value suggests the formation of condensed well-packed monolayer.

\section{Characterization of CQD}

We have characterized the size distribution of CQDs using TEM and DLS measurements as shown in Figure S1. Details of the TEM and DLS measurements have been given in the manuscript.

(a)

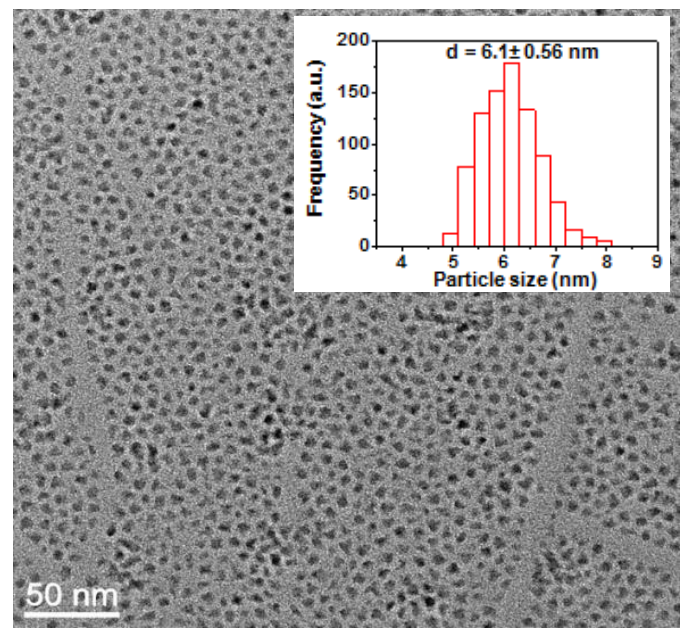

(b)

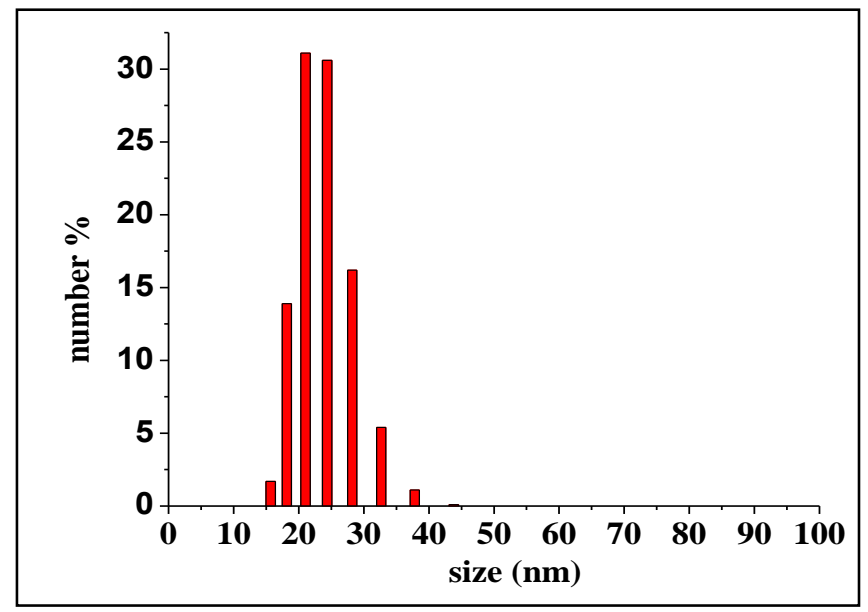

Figure S1: (a) TEM image (along with particle core size (d) distribution at inset) (b) dynamic light based hydrodynamic size distribution of CQDs. 


\section{X-ray Reflectivity Measurements:}

X-ray reflectivity (XR) measurements were conducted to understand out-of-the-plane structures of these two-component SLBs in detail. These experiments were performed at a synchrotron source. All the XR data from these SLBs were collected at Indian Beamline (BL-18B), Photon Factory, KEK, Japan. The X-ray energy of $16 \mathrm{keV}$ (corresponds to X-ray wavelength $=0.77 \AA$ ) was used with a beam of size of $0.15(\mathrm{~V}) \times 1.2(\mathrm{H}) \mathrm{mm}^{2}$. The scattered beam was collected by a NaI scintillation detector as a function of incident angle. To maintain complete hydration of the SLBs during the measurements, a homemade liquid cell was employed (Figure S2). We utilized the standard reflectivity software (IGOR with motofit package) to analyze the reflectivity curves. The reflectivity curves have been handled as arising from a stack of parallel layers having different thickness and scattering length density depending at the composition.

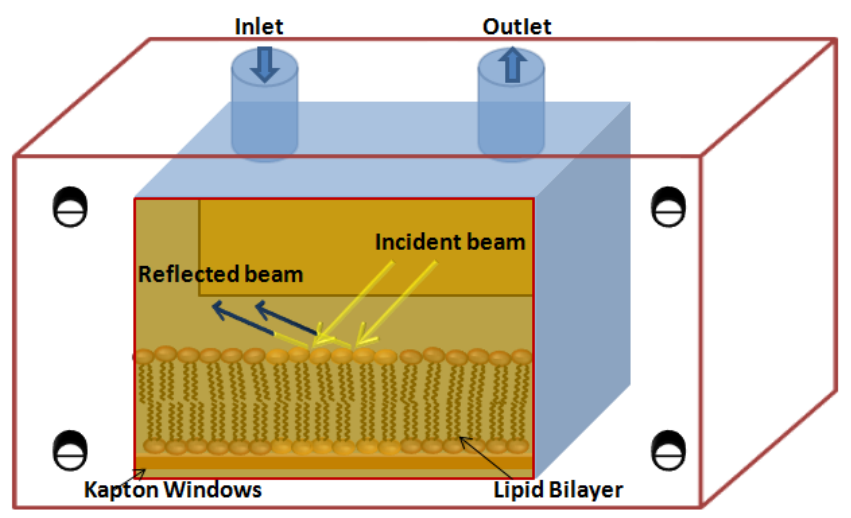

Figure S2: Schematic diagram of supported lipid bilayer deposited on silicon substrate along with the liquid cell used for X-ray reflectivity (XR) measurements. 
Different models have been used and compared to fit the experimental XR curves of pure SLBs having different compositions. In presence of CQDs an extra layer consisting of CQD core was taken into consideration. In order to minimize the number of fitting parameters, electron density of water and Si were fixed to 0.334 and $0.713 \mathrm{el} / \AA^{3}$ respectively. As a metric to quantify the goodness of fit for different models, we have tabulated the Chi squared values in table S1.

For P1G1, XR data were analyzed using Five and six-layered systems (Figure S3). The Fivelayered system comprises of (1) outer head groups $\left(\mathrm{head}_{2}\right)$ in contact with the PBS buffer, (2) outer tails (tail 2$),(3)$ inner tails (tail 1$)$, (4) inner head groups $\left(\right.$ head $\left._{1}\right)$ and (5) silicon dioxide layer.
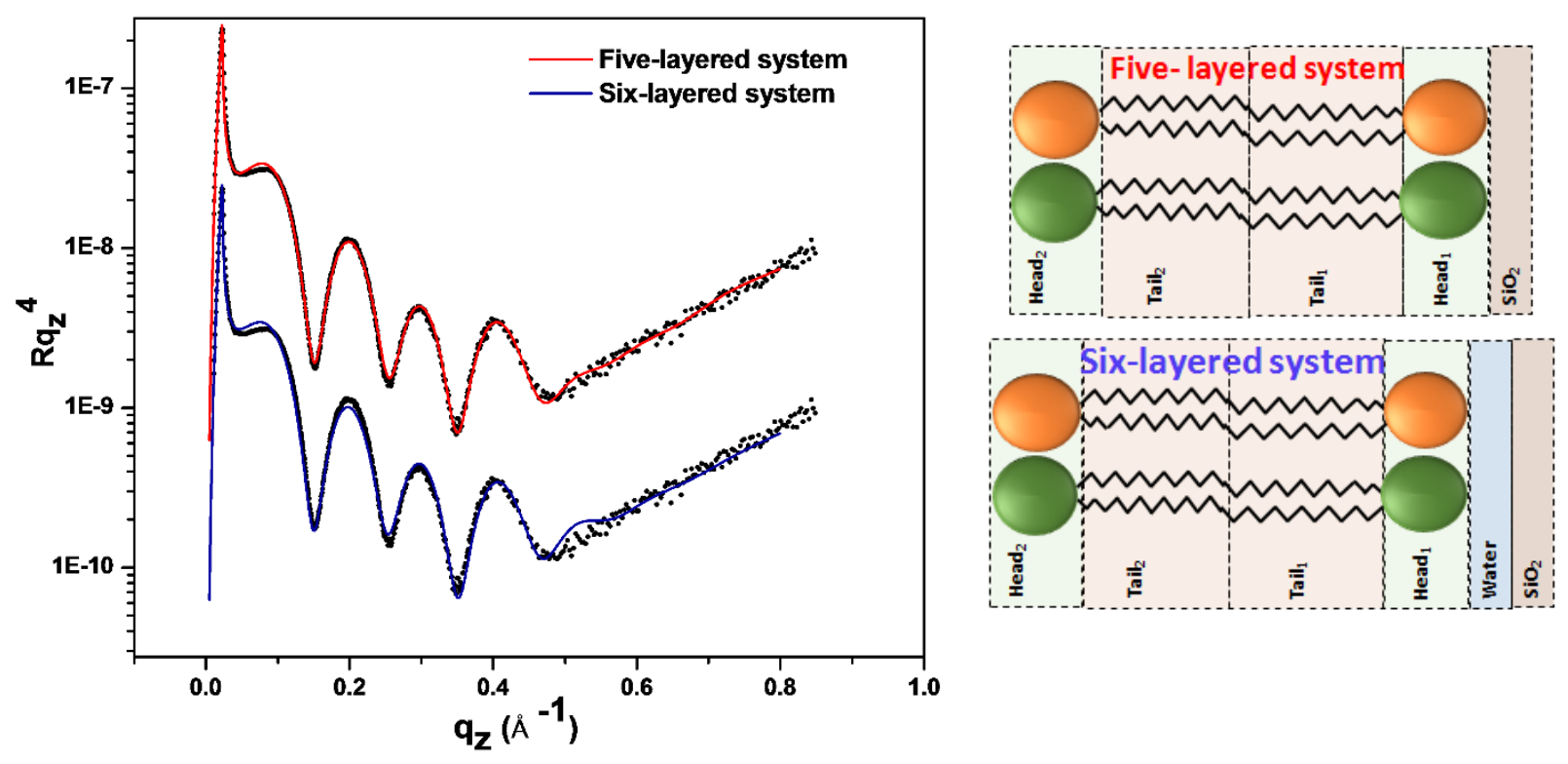

Figure S3: Different XR fitting systems used for the fitting of P1G1 SLBs data. Symbols correspond to the data and solid lines represent the fit. Reflectivity profiles are shifted vertically for clarity.

In case of six-layered model we have added an extra layer of water in between the inner head group and silicon dioxide layer. As shown in Figure S3, a five-layered system could satisfactorily fit the 
experimental curves and give physical explanation, therefore it was chosen for further studies related to the interaction of P1G1 with CQDs.

In case of M1G1, Five and two different six-layered systems (designated as 6A and 6B) were used (Figure S4). Five-layered system is same as described above for P1G1. 6A model is analogous in composition as that of Five-layered system, with an extra layer of water between head 1 and silicon dioxide. While 6B system contains (1) outer head groups $\left(\right.$ head $\left._{2}\right)$ in contact with the PBS buffer, (2) outer tails (tail 2 ), (3) $\mathrm{CH}_{3}$ group, (4) inner tails (tail 1 ), (5) inner head groups (head 1 ) and (6) silicon dioxide layer. In our study, the fit of M1G1 is based on a 6B system which is describing the best fit for XR data of M1G1 (Figure S4).
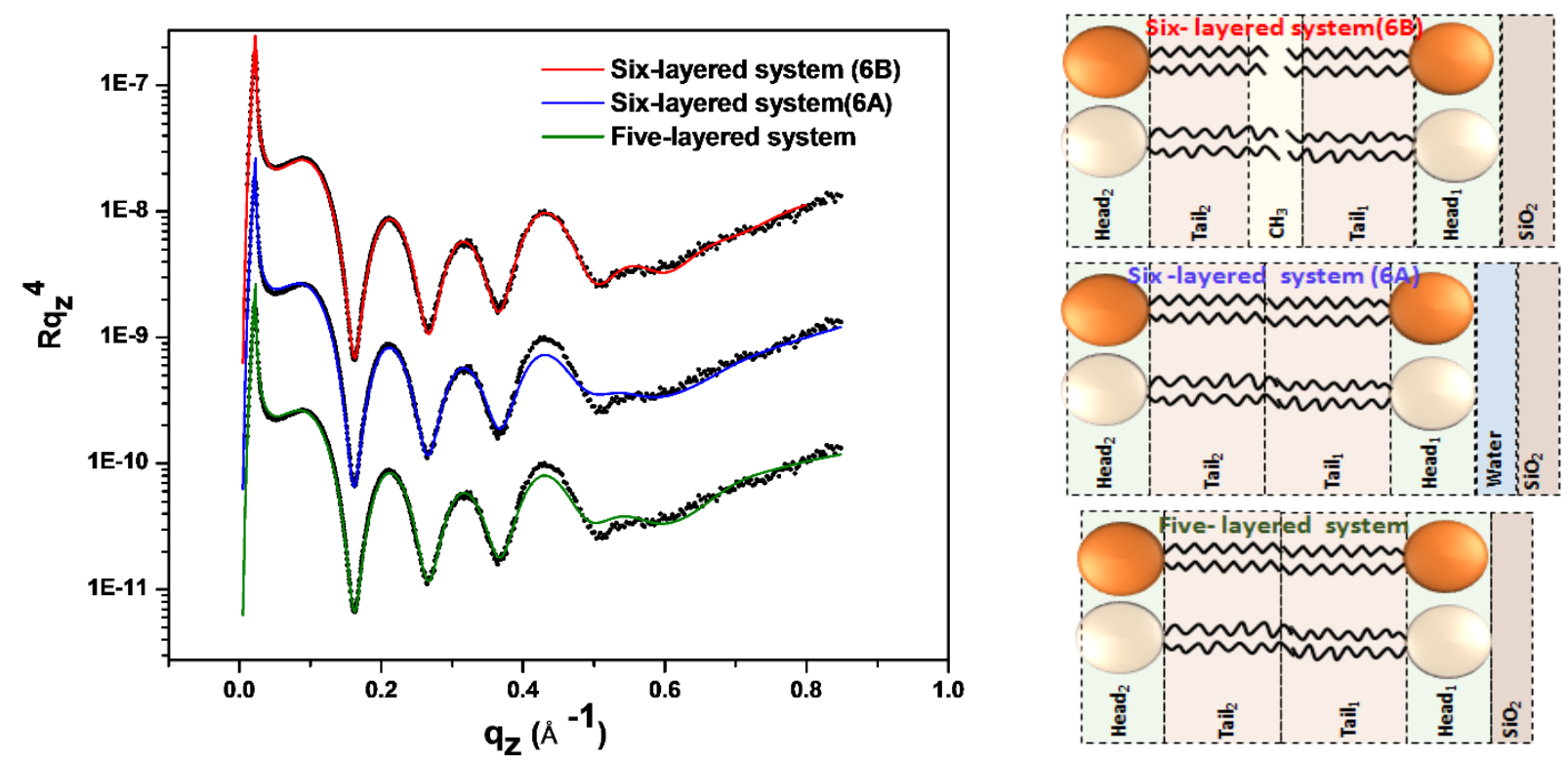

Figure S4: Different XR fitting systems used for the fitting of M1G1 SLBs data. Symbols correspond to the data and solid lines represent the fit. Reflectivity profiles are shifted vertically for clarity.

Five, Six and seven layered systems were used to fit O1G1 SLBs XR data. Five and six layers systems are same as described for P1G1 while in case of seven-layered systems, we split both 
upper and lower heads. It is clear from figure S5 that the fits from five and six layered systems were not as good as obtained from the seven-layered system. The latter is having (1) outer head groups of DPPG (head 2 b) in contact with the PBS buffer, (2) outer head groups of DOPC and outer tails (head ${ }_{2 \mathrm{a}}+$ tail $\left._{2}\right),(3)$ outer tails (tail 2$),(4)$ inner tails (tail 1$),(5)$ inner head groups of DPPG and inner tails (head $\left.{ }_{1 b}+t_{a i l}\right)$, (6) inner head groups of DOPC (head ${ }_{1 a)}$ and (7) silicon dioxide, respectively. The best fitting parameters for all these two-component SLBs i.e. thickness and electron density (ED) are summarized in Tables S1-S4.
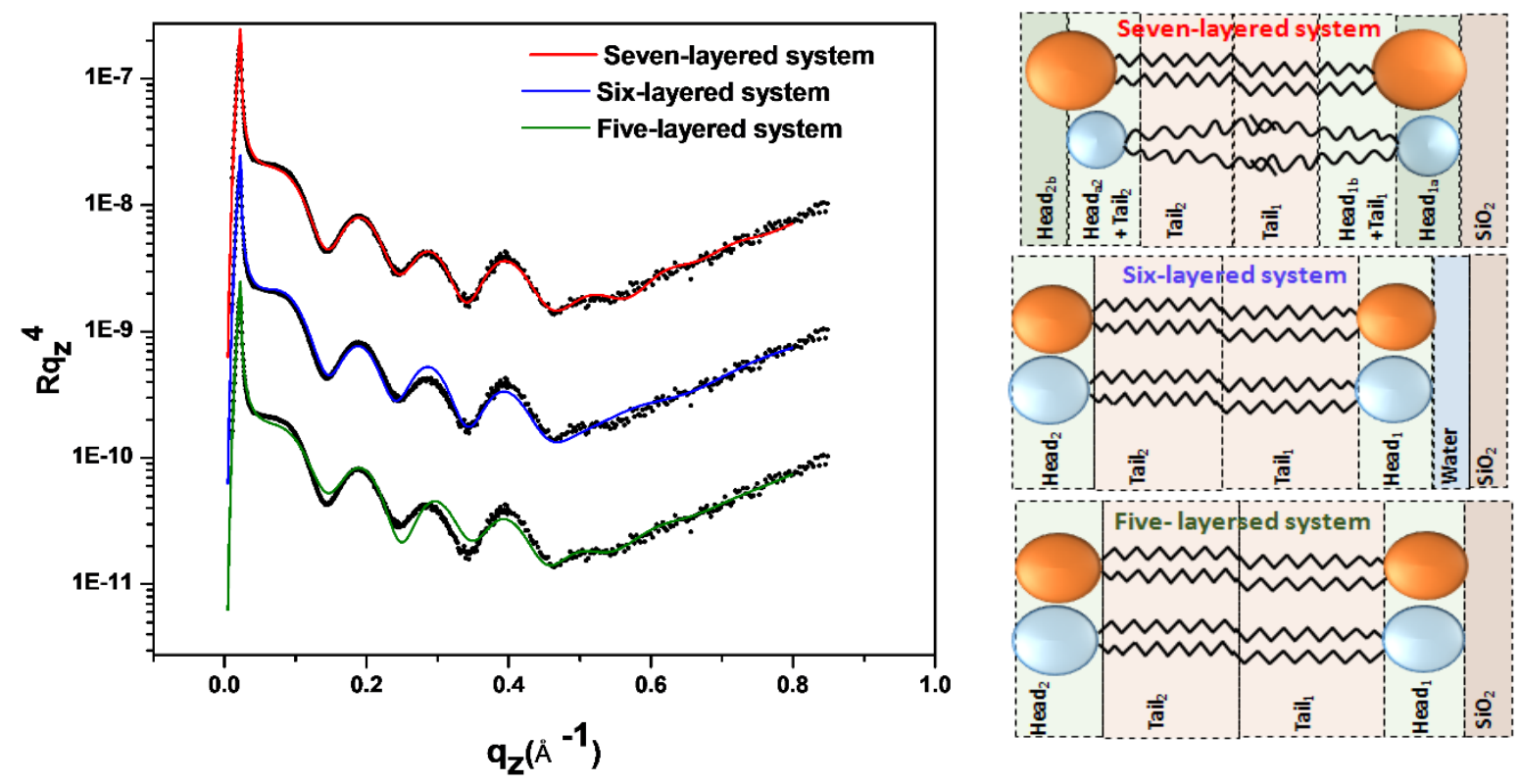

Figure S5: Different XR fitting systems used for the fitting of O1G1 SLBs data. Symbols correspond to the data and solid lines represent the fit. Reflectivity profiles are shifted vertically for clarity.

\section{Atomic force microscopy (AFM) Measurements:}

AFM imaging of O1G1 SLBs were performed under water using NT-MDT (Russia) System with a Pyrex-Nitride-Probes-Silicon Nitride (PNP-SiN) cantilever of force constant $0.32 \mathrm{~N} / \mathrm{m}$. We used 
contact mode imaging with a scan speed of $0.8 \mathrm{~Hz}$ for $9 \mu \mathrm{m}$ X $9 \mu \mathrm{m}$. The AFM images were collected from P1G1, M1G1 and O1G1 bilayers transferred on glass substrate at $32 \mathrm{mN} / \mathrm{m}$ at 20 ${ }^{\circ} \mathrm{C}$. A height difference of $\sim 1 \mathrm{~nm}$ between the $\mathrm{L}_{\mathrm{d}}$ domain (DOPC in both leaflets) and the ordered DPPG domain (DPPG in both leaflets) could be detected. However, in M1G1 and P1G1, no such height difference between the domains could be detected. The line profiles in (d), (e) show the heights of the P1G1 and M1G1 SLBs measures across a defect, whereas (f) shows the height across the $\mathrm{L}_{\mathrm{o}}-\mathrm{L}_{\mathrm{d}}$ domains in the O1G1 SLB (c).
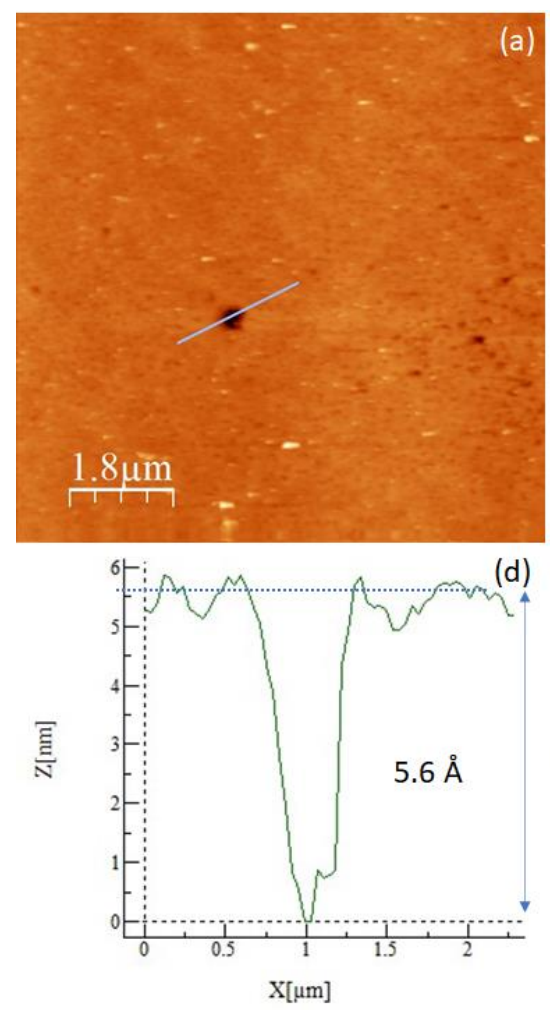

(d)
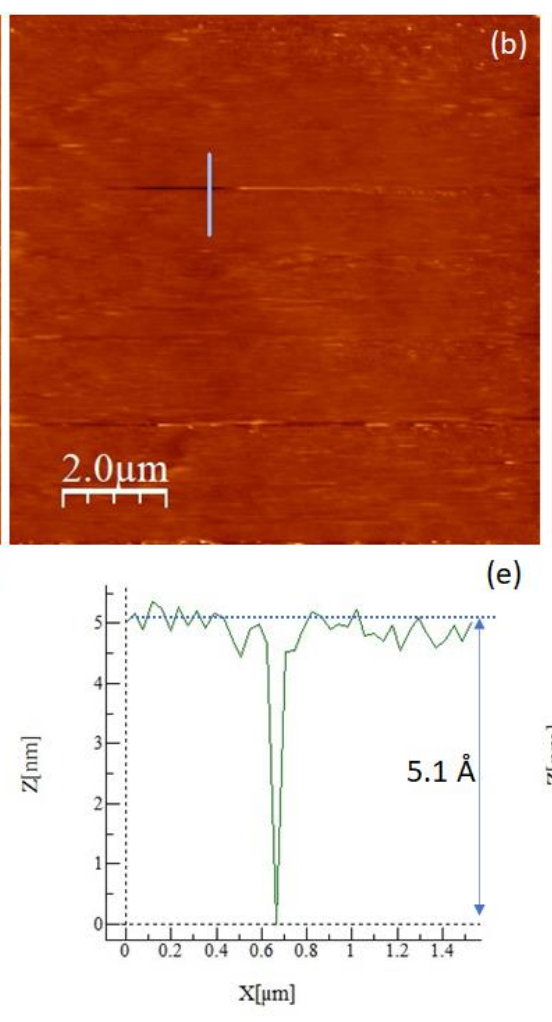
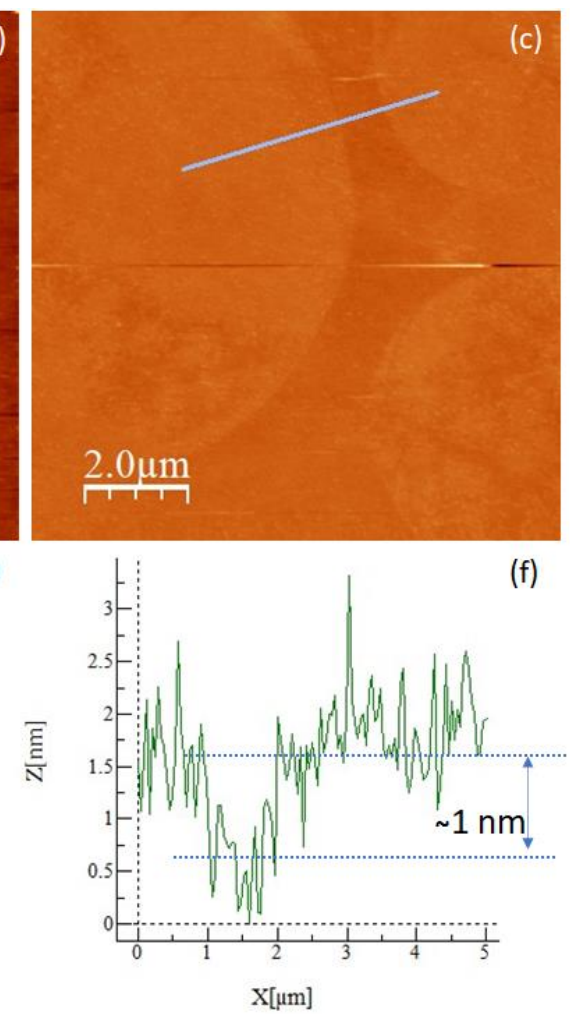

Figure S6: AFM images of (a) P1G1, (b) M1G1 and (c) O1G1 SLBs transferred on glass substrate by LB/LS method. (g)-(i) show the height across the line profile in respective images. 


\section{Confocal imaging:}

Confocal images were recorded using a commercial Microscopy setup (LEICA TCS SP5 II, GmbH, Mannheim, Germany) with 100X oil objective lens at $640 \mathrm{nM}$ excitation and analyzed by LAS AF microscopy software. To make the bilayer luminescent, dye-tagged lipid (Atto647DMPE, $0.05 \mathrm{~mol} \%$ ) was added in lipid mixture and mixed thoroughly before spreading at the airwater interface on a Langmuir trough. After transfer of the bilayer onto the glass coverslip using LB/LS technique (described in the Methods section) the bilayers were transferred to a container filled with buffer without exposing to air and stored at $25^{\circ} \mathrm{C}$ and used as such. For imaging, 512 $\times 512$ pixels at a $600 \mathrm{~Hz}$ frame rate was used for image acquisition. The images reveal phase separation between the domains in the bilayers. The nature of the domains could be readily identified from the O1G1 and M1G1 images. Although the confocal image of P1G1 undoubtedly pertains to phase separation, yet the uneven dye-partitioning, owing to very high isothermal compression modulus/rigidity of the membrane, do not clearly tell about the true nature of the domains.
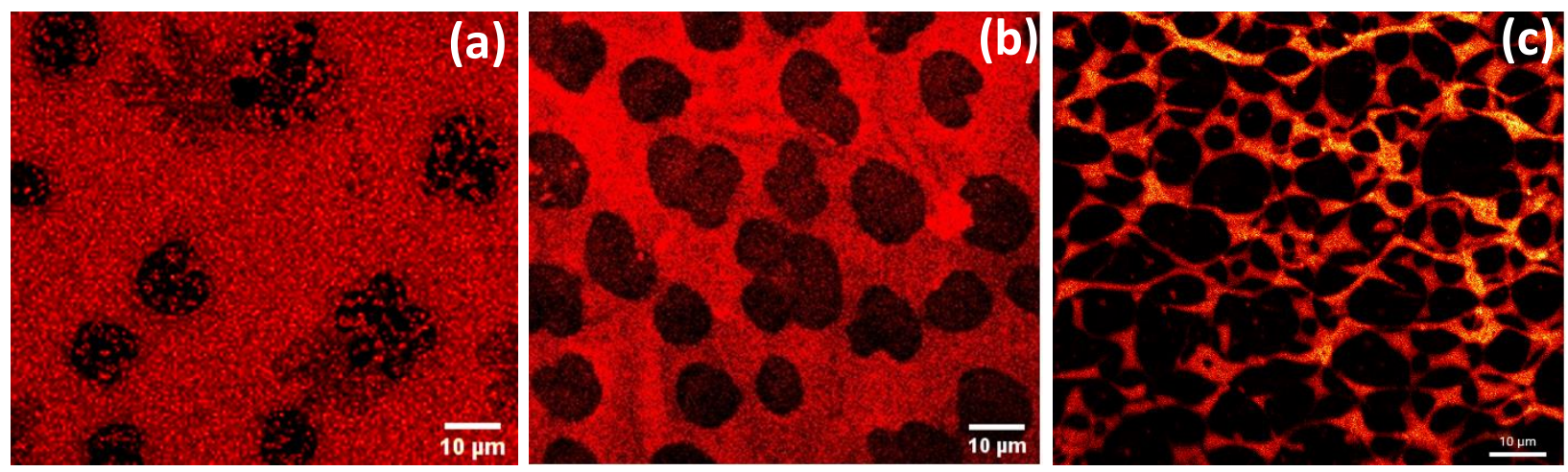

Figure S7: The confocal microscopy images of P1G1, M1G1 and O1G1 SLBs respectively (a-c). The lighter regions are dye rich and the darker region are dye poor region. All the bilayers are stained with Atto647N DMPE (0.05mol \%). 
Table S1: Chi squared values for different models.

\begin{tabular}{|c|c|}
\hline & $\chi^{2}$ \\
\hline P1G1 5-layered system & 47.89 \\
\hline P1G1 6-layered system & 157.04 \\
\hline P1G1+5nM CQD & 35.49 \\
\hline P1G1+ 5nM CQD_NO CQD layer & 48.62 \\
\hline P1G1+10nM CQD & 22 \\
\hline P1G1+10nM CQD_NO CQD layer & 217.75 \\
\hline & \\
\hline M1G1 5-layered system & 41.56 \\
\hline M1G1 6-layered system & 23.22 \\
\hline M1G1+ 5nM CQD & 23.46 \\
\hline M1G1+5nM CQD_NO CQD layer & 140.15 \\
\hline M1G1+ 10nM CQD & 17.08 \\
\hline M1G1+10nM CQD_NO CQD layer & 35.07 \\
\hline O1G1 5-layered system & \\
\hline O1G1 6-layered system & 71 \\
\hline O1G1 7-layered system & 62 \\
\hline O1G1+5nM CQD & 32 \\
\hline O1G1+ 5nM CQD_NO CQD layer & 35.24 \\
\hline O1G1+ 10nM CQD & 43.98 \\
\hline O1G1+10nM CQD_NO CQD layer & 99.98 \\
\hline & \\
\hline O1G1+ 5nM CQD_NO CQD layer_pH5.9 & 20.46 \\
\hline & 90.72 \\
\hline
\end{tabular}


Table S2: Parameters obtained from the fits of X-ray reflectivity data for P1G1 at $\mathrm{pH} 7 . \mathrm{t}_{\mathrm{n}}, \rho_{\mathrm{n}}$ denote the thickness and electron density of the different layers.

\begin{tabular}{|c|c|c|c|c|c|c|}
\hline \multirow[t]{2}{*}{ layer } & \multicolumn{2}{|c|}{ Only P1G1 } & \multicolumn{2}{|c|}{ P1G1+5nM CQD } & \multicolumn{2}{|c|}{ P1G1+10nM CQD } \\
\hline & $\operatorname{tn}(\AA)$ & $\rho_{n}\left(e l / \AA^{3}\right)$ & $\operatorname{tn}(\AA)$ & $\rho_{n}\left(e l / \AA^{3}\right)$ & $\operatorname{tn}(\AA)$ & $\rho_{n}\left(e l / \AA^{3}\right)$ \\
\hline Water & INF & 0.334 & INF & 0.334 & INF & 0.334 \\
\hline CQD & ---- & ---- & 14.87 & 0.344 & 13.29 & 0.363 \\
\hline head 2 & 9.77 & 0.439 & 9.55 & 0.436 & 9.19 & 0.327 \\
\hline tail 2 & 18.94 & 0.283 & 17.42 & 0.301 & 14.09 & 0.284 \\
\hline tail 1 & 20.84 & 0.266 & 20.77 & 0.292 & 15.81 & 0.297 \\
\hline head1 & 9.95 & 0.448 & 9.89 & 0.447 & 9.55 & 0.411 \\
\hline $\mathrm{SiO}_{2}$ & 5.98 & 0.656 & 5.98 & 0.656 & 5.98 & 0.656 \\
\hline $\mathbf{S i}$ & INF & 0.713 & INF & 0.713 & INF & 0.713 \\
\hline
\end{tabular}

Table S3: Parameters obtained from the fits of X-ray reflectivity data for M1G1at $\mathrm{pH}$ 7. $\mathrm{t}_{\mathrm{n}}, \rho_{\mathrm{n}}$ denote the thickness and electron density of the different layers.

\begin{tabular}{|c|c|c|c|c|c|c|}
\hline \multirow[t]{2}{*}{ layer } & \multicolumn{2}{|c|}{ Only M1G1 } & \multicolumn{2}{|c|}{ M1G1+ 5nM CQD } & \multicolumn{2}{|c|}{ M1G1+10nM CQD } \\
\hline & $\operatorname{tn}_{\mathbf{n}}(\AA)$ & $\rho_{n}\left(e l / \AA^{3}\right)$ & $\operatorname{tn}(\AA)$ & $\rho_{n}\left(e l / \AA^{3}\right)$ & $\operatorname{tn}(\AA)$ & $\rho_{n}\left(\mathrm{el} / \AA^{3}\right)$ \\
\hline Water & INF & 0.334 & INF & 0.334 & INF & 0.334 \\
\hline CQD & $\begin{array}{l}---- \\
\end{array}$ & ---- & 9.63 & 0.339 & 13.04 & 0.343 \\
\hline head2 & 9.77 & 0.431 & 9.44 & 0.402 & 8.79 & 0.328 \\
\hline tail 2 & 17.19 & 0.318 & 15.17 & 0.314 & 10.01 & 0.321 \\
\hline $\mathrm{CH}_{3}$ & 1.34 & 0.207 & 5.56 & 0.199 & 5.54 & 0.330 \\
\hline tail1 & 17.99 & 0.312 & 15.78 & 0.316 & 11.16 & 0.335 \\
\hline head1 $_{1}$ & 10.55 & 0.453 & 10.06 & 0.420 & 10.09 & 0.429 \\
\hline $\mathrm{SiO}_{2}$ & 5.69 & 0.656 & 5.69 & 0.656 & 5.69 & 0.656 \\
\hline $\mathbf{S i}$ & INF & 0.713 & INF & 0.713 & INF & 0.713 \\
\hline
\end{tabular}


Table S4: Parameters obtained from the fits of X-ray reflectivity data for O1G1 pH7. $t_{n}, \rho_{n}$ denote the thickness and electron density of the different layers.

\begin{tabular}{|c|c|c|c|c|c|c|}
\hline \multirow[t]{2}{*}{ layer } & \multicolumn{2}{|c|}{ Only O1G1 } & \multicolumn{2}{|c|}{ O1G1+5nM CQD } & \multicolumn{2}{|c|}{ O1G1+10nM CQD } \\
\hline & $\operatorname{tn}(\AA)$ & $\rho_{n}\left(e l / \AA^{3}\right)$ & $\operatorname{tn}(\AA)$ & $\rho_{n}\left(e l / \AA^{3}\right)$ & $\operatorname{tn}(\AA)$ & on $\left(\mathrm{el} / \AA^{\AA^{3}}\right)$ \\
\hline Water & INF & 0.334 & INF & 0.334 & INF & 0.334 \\
\hline lipidcomplex & ---- & ---- & ---- & $\begin{array}{ll}--- \\
\end{array}$ & 17.68 & 0.314 \\
\hline CQD & ---- & ---- & 10.68 & 0.33605 & ---- & ---- \\
\hline head $2 b$ & 5.89 & 0.394 & 6.34 & 0.371 & 4.49 & 0.325 \\
\hline head2a +tail 2 & 7.86 & 0.426 & 8.58 & 0.379 & 4.11 & 0.328 \\
\hline tail 2 & 12.70 & 0.216 & 12.88 & 0.176 & 7.12 & 0.298 \\
\hline tail1 & 13.50 & 0.311 & 12.81 & 0.316 & 7.92 & 0.270 \\
\hline head $_{1 b}+$ tail $_{1}$ & 5.22 & 0.462 & 5.54 & 0.454 & 3.52 & 0.296 \\
\hline head1a $_{1}$ & 8.35 & 0.440 & 8.51 & 0.455 & 5.10 & 0.410 \\
\hline $\mathrm{SiO}_{2}$ & 14 & 0.649 & 14 & 0.649 & 14 & 0.649 \\
\hline $\mathbf{S i}$ & INF & 0.713 & INF & 0.713 & INF & 0.713 \\
\hline
\end{tabular}

Table S5: Parameters obtained from the fits of X-ray reflectivity data for O1G1at $\mathrm{pH}$ 5.9. $\mathrm{t}_{\mathrm{n}}, \rho_{\mathrm{n}}$ denote the thickness and electron density of the different layers.

\begin{tabular}{|c|c|c|c|c|}
\hline \multirow[t]{2}{*}{ layer } & \multicolumn{2}{|c|}{ Only O1G1 } & \multicolumn{2}{|c|}{ O1G1+5nM CQD } \\
\hline & $\operatorname{tn}(\AA)$ & $\rho_{n}\left(e l / \AA^{3}\right)$ & $\operatorname{tn}(\AA)$ & $\rho_{n}\left(e l / \AA^{3}\right)$ \\
\hline Water & INF & 0.334 & INF & 0.334 \\
\hline CQD & $\begin{array}{l}--- \\
\end{array}$ & $\begin{array}{l}--- \\
\end{array}$ & 16.83 & 0.350 \\
\hline head2b & 5.31 & 0.412 & 4.77 & 0.328 \\
\hline head2a +tail 2 & 7.48 & 0.450 & 6.29 & 0.326 \\
\hline tail2 & 11.03 & 0.167 & 10.45 & 0.303 \\
\hline tail1 & 11.72 & 0.245 & 10.91 & 0.277 \\
\hline head $_{1 b}+$ tail $_{1}$ & 5.46 & 0.417 & 4.93 & 0.258 \\
\hline head1a & 7.56 & 0.438 & 5.51 & 0.408 \\
\hline $\mathrm{SiO}_{2}$ & 10.81 & 0.639 & 10.81 & 0.639 \\
\hline Si & INF & 0.713 & INF & 0.713 \\
\hline
\end{tabular}

